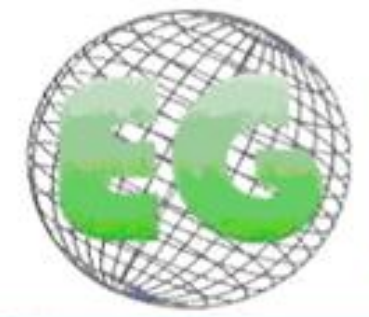

$N^{\circ} 37$

www.um.es/egloball

\title{
Métodos anticonceptivos en las mujeres inmigrantes y el papel de la enfermera: una revisión bibliográfica
}

Contraception in immigrant women and the role of the nurse: a literature review

\section{*Solana Morete, Álvaro **González López, José Rafael}

\author{
"Graduado en Enfermería. Universidad de Sevilla "*Doctor Internacional en Ciencias de la Salud por \\ la Universidad de Sevilla. Profesor de la Facultad de Enfermería, Fisioterapia y Podología. \\ Departamento de Enfermería de la Universidad de Sevilla, España E-mail: joserafael@us.es
}

Palabras Clave: Migración Internacional; Anticoncepción; Planificación Familiar; Salud de la Mujer; Cuidados de Enfermería.

Keywords: Emigration and Immigration; Contraception; Contraceptive; Family Planning; Women's Health; Nursing care

\section{RESUMEN}

Introducción: En España en el año 2013 había un total de 6.259 .137 extranjeros, el 49\% eran mujeres, esto da lugar a que la salud reproductiva de este colectivo suponga una preocupación importante en el campo de la Salud Pública en España, ya que es uno de los grupos más demandantes de determinados servicios sanitarios.

Objetivo: Describir la situación actual del empleo de métodos anticonceptivos de la mujer inmigrante en España en los últimos años (2005 en adelante).

Metodología: Se realizó una revisión bibliográfica en la primera quincena de marzo de 2014, se limitó a artículos y revisiones bibliográficas publicadas a partir del 2005 en inglés y español. Las bases de datos utilizadas fueron PubMed, IME, SCOPUS, CINAHL, CUIDEN, WOS, Cochrane Library y TESEO.

Resultados: La enfermera juega un papel fundamental en el conocimiento y uso de nuevos métodos anticonceptivos por parte de la mujer inmigrante, contando con que el DIU es más utilizado por la inmigrante magrebí y de Europa del Este, el preservativo es el más usado por la inmigrante latinoamericana, la asiática conoce bien todos los métodos pero prefiere la medicina tradicional china, y la subsahariana es la que tiene menor índice de uso de métodos anticonceptivos.

Conclusiones: Existe una variabilidad de métodos anticonceptivos por parte de la mujer inmigrante en España según su religión y/o cultura. El profesional de Enfermería, debe conocer dichas peculiaridades, con el fin de ofrecer un cuidado transcultural a la paciente y su pareja al objeto de favorecer una planificación familiar efectiva. 


\section{ABSTRACT}

Introduction: There were a total of $6,259,137$ foreigners in Spain in $2013,49 \%$ of which were women. This leads to the reproductive health of this collective supposing an important problem in the field of Public Health in Spain, since it is one of the most demanding groups in certain health services. Objective: To describe the current situation of contraceptive methods of immigrant women in Spain in recent years (2005 onwards).

Methodology: A literature review was conducted in the first half of March 2014, it was limited to articles and literature reviews published since 2005 in English and Spanish. The databases used were PubMed, IME, SCOPUS, CINAHL, CUIDEN, WOS, Cochrane Library and TESEO databases.

Results: The nurse plays a key role in the knowledge and use of new contraceptive methods by immigrant women, with the IUD being used by Maghrebi and Eastern European immigrants, the condom is the most used by the Latino immigrants. Asians know all the methods but prefer the traditional Chinese medicine, while the Sub-Saharan is the one who has the lowest rates of contraceptive use.

Conclusions: The variability of contraceptive methods used by immigrant women in Spain depends on their religion and/or culture. The nursing professional must know these peculiarities, in order to provide a transcultural care to the patient and her partner in order to promote effective family planning.

\section{INTRODUCCIÓN}

El hecho migratorio ha constituido un fenómeno constante en la naturaleza social de la Humanidad. Países y continentes se han desarrollado gracias a dicho fenómeno, siendo el contacto con otras culturas lo que ha conformado la base de sus identidades $^{(1)}$. España es uno de los destinos elegidos por los inmigrantes a la hora de emigrar ${ }^{(2)}$. Los inmigrantes provenientes fundamentalmente de América latina, el Magreb, el África subsahariana, Europa del Este y Asia central han aumentado en los últimos años, siendo un hecho reseñable la feminización de la población migrante ${ }^{(3)}$. Durante la primera década del siglo XXI, los inmigrantes en este país pasaron de ser el $1,8 \%$ del total de la población residente en España al 12,2\%; según la procedencia el $40 \%$ de los extranjeros provenía de Europa, siguiéndole con un 31\% América del Sur ${ }^{(3-5)}$. En el año 2013 había en España un total de 6.259.137 extranjeros, hombres y mujeres residentes, según datos del Ministerio de Trabajo y Asuntos Sociales, de ellos, el $49 \%$ eran mujeres ${ }^{(6)}$.

Según el país de procedencia, varía por ende la cultura, sus costumbres y concepciones sobre el binomio salud-enfermedad, entre otros. Dichos factores influyen a la hora de atender a estas poblaciones y su participación del Sistema Sanitario Nacional. Las mujeres inmigrantes tienen una serie de características que comparten, pese a su procedencia; es reseñable el bajo nivel educativo, mayor riesgo de problemas de salud de la esfera psíquica (depresiones, ansiedad), menor asistencia a los servicios sanitarios debido a su desconocimiento de los mismos y son más vulnerables a sufrir violencia de género ${ }^{(7)}$.

Debido a la feminización de la población inmigrante y a las diferentes concepciones de la salud sexual y reproductiva, el empleo de métodos anticonceptivos se ve influenciado por el país de origen de los inmigrantes ${ }^{(3)}$. Desde la disciplina enfermera es importante saber qué métodos anticonceptivos conocen, y si utilizan alguno, para así poder hacer recomendaciones sobre su fiabilidad y uso; sabiendo que es fundamental informar adecuadamente para su uso correcto o la mejora del cumplimiento de éste, así como detectar situaciones de riesgo. En definitiva, la 
educación sanitaria es un pilar importante para la planificación familiar, teniendo en cuenta la transculturalidad de los cuidados ${ }^{(7)}$.

Debido a que en la última década ha existido un constante incremento de la inmigración, y ésta ha sido importante tanto en diversidad de procedencias como en número, están apareciendo diferentes necesidades de formación e información en todos los agentes sociales, y muy especialmente en los profesionales de la salud, que son el primer contacto con nuestro sistema sanitario y gestores del paciente por parte de esta población. Todos estos cambios sociológicos nos exigen el aprendizaje de nuevas habilidades, destrezas y conocimientos, a la vez que requieren un cambio en nuestras aptitudes y actitudes ${ }^{(8)}$.

En general, estamos hablando de poblaciones que se componen en su mayoría por personas adultas jóvenes en edad reproductiva. El hecho de la feminización de la población inmigrante y de la caracterización demográfica expuesta con anterioridad, está dominando los flujos migratorios en España, lo que asegura la supervivencia del colectivo y por consiguiente el surgimiento de nuevas necesidades en materia de salud reproductiva, las cuales han de ser contempladas de diferentes formas según el país de origen, cultura, tradiciones, ritos; por lo que las necesidades de cuidados han de ser consideradas desde un punto de vista multicultural o transcultural ${ }^{(3)}$.

En el caso de las mujeres que emigran de su país origen y llegan a España, a pesar de ser un grupo heterogéneo, parece que todas tienen en común multitud de factores que las convierten en un colectivo especialmente "vulnerable" a sufrir embarazos no deseados e interrupciones de los mismos con el consiguiente coste biopsicosocial que supone para ellas. El problema básico al que nos enfrentamos es determinar hasta que punto el sistema sanitario, los programas y los profesionales tienen en cuenta las creencias culturales que influyen en el concepto de salud que poseen los distintos colectivos de mujeres inmigrantes que conviven en el país, es decir, hasta que punto comprenden qué se entiende por salud en cada cultura y su consistencia hereditaria $^{(7)}$.

A pesar de que España cuenta con un Sistema Nacional de Salud de cobertura universal, y que otorga a los extranjeros empadronados el derecho a las prestaciones sanitarias en las mismas condiciones que a la población autóctona, y en el caso de los no empadronados la atención urgente, la materno e infantil hasta los dieciocho años, como refleja el Real Decreto-Ley 16/2012, de 20 de abril ${ }^{(8)}$; se han evidenciado desigualdades entre la población autóctona y la inmigrante en relación a la salud percibida, no explicadas exclusivamente por la condición socioeconómica y que podrían estar apuntando a diferencias en el acceso a la atención en salud ${ }^{(9)}$. Entre los factores que generan estas desigualdades en el acceso en nuestro entorno y en los países con más tradición migratoria y sistemas de salud semejantes, se encuentran además de las condiciones socioeconómicas, las barreras idiomáticas, culturales y la inadaptabilidad de los servicios a las necesidades específicas de esta población ${ }^{(10)}$. En lo que respecta a la salud sexual y reproductiva es frecuente en la población inmigrante el desconocimiento de los distintos métodos anticonceptivos existentes. Siendo algunas culturas reacias a su utilización por muy diferentes motivos, como son la sobrevaloración de la fertilidad o la falta de experiencia en el uso de algún método; mientras otros colectivos de inmigrantes sí que van a solicitar este tipo de métodos ${ }^{(3)}$.

Con esta revisión bibliográfica, se pretende describir la situación actual del empleo de métodos anticonceptivos de la mujer inmigrante en España en los últimos años, desde 
el año 2005, para actualizar los conocimientos sobre la misma, y poder así abrir nuevas líneas de investigación destinadas a las enfermeras para mejorar el trato con la mujer inmigrante y reducir costes sanitarios.

\section{MATERIAL Y MÉTODO}

Se diseñó una revisión bibliográfica en la que se consultaron diferentes bases de datos, tales como: PubMed, IME, SCOPUS, CINAHL, CUIDEN, WOS y Cochrane Library. Se utilizaron descriptores como "Emigrants and Immigrants"[Mesh] AND "Contraception"[Mesh], "contraception AND contraceptive method AND sociocultural aspect", "inmigra*" $Y$ "anticoncep*", "immigrants AND contraceptive methods", "nurse AND contraceptive methods", "immigrants AND contraceptive", "enfermer" "transcultural" y "transculturalidad", tal y como puede apreciarse en la Tabla 1. La búsqueda abarcó el periodo de tiempo desde 2005 a marzo de 2014.

El ámbito de este trabajo se centra en los métodos anticonceptivos en las mujeres inmigrantes en España. Se descartaron muchos artículos al ser estudios de otros países que no aportaban nada a esta revisión al centrarse en la violencia de género y otros al ser repetición de artículos ya encontrados con anterioridad en otra base de datos consultada. Por último, se han tenido en cuenta las referencias bibliográficas secundarias y los artículos recomendados de los artículos encontrados.

Una vez revisados los resúmenes de todas las referencias encontradas, cada autor discriminó aquellas que podían relacionarse con el objetivo de la búsqueda, de un total de 215 artículos encontrados, se seleccionaron 25. Las controversias fueron resueltas por acuerdo entre los autores. Para seleccionar la evidencia se tuvieron en cuenta los resúmenes, el año de publicación teniendo que ser a partir del 2005, artículos relacionados con los artículos que se encontraron.

Tabla 1. Bases de datos que se han empleado con los descriptores empleados para las búsquedas.

\begin{tabular}{|c|c|}
\hline Base datos & Descriptores \\
\hline PubMed & $\begin{array}{c}\text { Emigrants and Immigrants"[Mesh] AND } \\
\text { "Contraception"[Mesh]; contraception AND } \\
\text { contraceptive method AND sociocultural aspect }\end{array}$ \\
\hline $\begin{array}{c}\text { IME (Índice Médico } \\
\text { Español) }\end{array}$ & "inmigra*" Y "anticoncep"” \\
\hline SCOPUS & $\begin{array}{c}\text { "immigrants AND contraceptive methods"; } \\
\text { "nurse AND contraceptive methods" }\end{array}$ \\
\hline $\begin{array}{c}\text { CINAHL (Cumulative } \\
\text { Index to Nursing and } \\
\text { Allied Health } \\
\text { Literature) }\end{array}$ & "immigrants AND contraceptive"; "enfermer"” \\
"transcultural"
\end{tabular}




\begin{tabular}{|c|c|}
\hline WOS (Web Of Science) & "inmigrant ${ }^{\star}$ Y anticoncep ${ }^{\star \prime}$ \\
\hline Cochrane Library & "immigrant AND contraception", "immigrant \\
contracep" AND
\end{tabular}

\section{RESULTADOS}

A continuación describiremos los resultados encontrados en la revisión bibliográfica en función de la región de procedencia de las mujeres.

\section{Latinoamérica}

Las mujeres latinoamericanas constituyen el colectivo de mujeres inmigrantes más numeroso en España ${ }^{(7)}$, tiene 2 características muy distinguidas, en primer lugar se trata de una inmigración discretamente feminizada (56\% en promedio en 2013 ) ${ }^{(11)}$ siendo ellas las que inician la aventura migratoria solas. En segundo lugar, suelen ocupar empleos en servicios desvalorizados socialmente (empleadas de hogar, cuidado de niños o ancianos, servicios sexuales), más de la mitad de ellas se encuentran en situación irregular. En 2005 antes de la última regularización, se contabilizó en situación irregular al $88 \%$ de bolivianos, $63 \%$ de argentinos, $55 \%$ de ecuatorianos, $50 \%$ de colombianos, $25 \%$ de dominicanos y $16 \%$ de peruanos. Estas cifras justificaron el proceso de regularización de dicho año ${ }^{(12)}$. En definitiva el perfil encontrado por un estudio ${ }^{(13)}$ sobre la mujer latinoamericana que trabaja es: eficacia a niveles laboral y social, ahorradoras, organizadas y constantes en el envío de dinero a su familia. Tienden a arreglar rápidamente su situación burocrática y reagrupar a su familia. Las familias españolas las prefieren por la igualdad idiomática y cierta afinidad cultural ${ }^{(13)}$. Existen altos índices de abortos debido a las prácticas de riesgo, así como la transmisión de infecciones de transmisión sexual (ITS) debido a la promiscuidad que tienen estas inmigrantes proveniente ya de sus países de origen, esta promiscuidad también provoca que gran parte de estas inmigrantes tengan varios hijos de padres diferentes, que dejan al cuidado de sus familiares ${ }^{(4,14)}$. La mayor parte de estas inmigrantes son mujeres jóvenes sexualmente activas (a partir de los 15-16 años) que no están familiarizadas ni acostumbradas a utilizar métodos anticonceptivos modernos. Existen discrepancias pero por lo general no saben cómo funciona un Sistema de Salud universal y por razones culturales prefieren acudir directamente al especialista, lo que explica ciertos comportamientos al llegar a España. Debido a que no conocen bien la legislación española y en sus países el aborto es ilegal, si desean interrumpir la gestación, recurren a métodos "naturales" y agresivos ${ }^{(4,7)}$.

Los métodos anticonceptivos que utilizan las mujeres solteras de estos países son insuficientes ya que los índices de infecciones de transmisión sexual son altos en la población joven, siendo el preservativo el método anticonceptivo menos usado en los jóvenes que mantienen relaciones sexuales por primera vez ${ }^{(15)}$. Los anticonceptivos orales son conocidos pero los utilizan mal, sin continuidad. También conocen los anticonceptivos parenterales debido a su uso extendido en Latinoamérica. Pueden acudir con DIU insertados en sus países conteniendo principios de cobre "T de cobre", en desuso en nuestro país, que pueden provocar hemorragias, dismenorrea y dificultades para su extracción. 
Ha aumentado la demanda de la píldora postcoital y despiertan su interés los implantes subdérmicos. Además se está produciendo un incremento de las IVEs (interrupciones voluntarias de embarazo) ${ }^{(7)}$. El método anticonceptivo que se utiliza con mayor frecuencia en esta población es el preservativo, seguido de los contraceptivos orales, y el DIU (16). El informe publicado durante el 2012 por el Ministerio de Sanidad sobre la IVE en España muestra datos sobre el lugar de residencia y nacimiento de la mujer. En España en este año hubo un total de 110.349 de IVES de los cuales, el 62\% (68.902 casos) correspondían a mujeres nacidas y residentes en España, frente al 38\% (41.447 casos) de mujeres procedentes de otros países y residentes en España. Según el reparto de continentes 18.770 IVES se produjeron en mujeres procedentes de América del Sur ${ }^{(6,17)}$.

\section{Magreb}

Su vida familiar está condicionada por la tradición, la religión, su cultura, la educación de los hijos, su alimentación, los hábitos higiénicos y la forma de vestir. Se integran y adaptan con dificultad a la sociedad de acogida, ya que existe una aceptación y una interiorización muy fuerte de las normas socio-tradicionales y religiosas de su país de origen $^{(4)}$. La Mudawana, o código de familia tradicional, regula las relaciones entre los miembros de la familia, teniendo el marido y el resto de familiares masculinos preeminencia absoluta, y ello a pesar de que desde 2004 rige en Marruecos un "Código de familia" que teóricamente consagra la igualdad de mujeres y hombres en el matrimonio ${ }^{(4)}$. En la cultura árabe, las cuestiones de las mujeres no se comparten con los hombres, además de tener un gran valor la virginidad prematrimonial.

Las mujeres magrebíes muestran mucho pudor ante los hombres, lo que puede causar problemas desde la primera visita. Por esto es conveniente tener el consentimiento del marido y realizar la exploración siempre que se pueda en presencia de otra mujer. Todo ello provocará un retraso en las actividades preventivas y en la atención de los problemas ${ }^{(7)}$. Los procedimientos anticonceptivos modernos eran tabú hasta hace poco tiempo para estas mujeres, y aún ahora en algunos países para que se les dispense un anticonceptivo deben demostrar que están casadas. Debido a que la menstruación es percibida como un tabú son mejor aceptados los métodos que acortan la menstruación (anticonceptivos hormonales), que los que la incrementan (DIU). Recurren al aborto con métodos peligrosos y tradicionales (4). Evolucionando la media de hijos por mujer de 7 (1970) a 3 (2005) en todos los países del Magreb ${ }^{(4,7)}$.

Según un estudio realizado en Chefchaouen en 2011 los métodos más conocidos entre las mujeres encuestadas son, el método ogino $(44,30 \%)$, el preservativo un $26,16 \%$, la píldora un $86,50 \%$ del total, el DIU (59,49\%) y el diafragma un $24,05 \%{ }^{(18)}$. Están prohibidas la ligadura de trompas y la vasectomía, son "haram" (ilícito). Sin embargo, en Marruecos el $50 \%$ de las mujeres casadas toman anticonceptivos orales, un $4 \%$ están esterilizadas y un $1,4 \%$ utilizan preservativos. Pese a sus ideas religiosas, la elección del método es variada, cuando acuden a nuestros centros; optando con frecuencia por DIU o anticonceptivos orales por el temor a quedarse de nuevo embarazadas ${ }^{(7)}$.

\section{África subsahariana}

Estas mujeres provienen de sociedades donde el respeto que una mujer recibe depende en gran medida del número de hijos. En África del Oeste y Central se encuentran las tasas de mortalidad materna más elevadas del mundo, varían de 500 
a 1.000 muertes maternas por 100.000 nacidos vivos, frente a la mortalidad de 1 a 10 por 100.000 nacidos vivos en los países desarrollados ${ }^{(7)}$. Por lo general la mujer tiene un estatus inferior al del hombre. Sobre ella recae el trabajo, el cuidado de la familia, de la casa y de los animales, así como conseguir comida y agua. En vez de la familia nuclear occidental, están acostumbradas a la familia clánica. Los matrimonios son muy precoces, lo cual condiciona que el $50 \%$ de las primigestas tengan menos de 14 años. En algunas culturas es frecuente la poligamia ${ }^{(4)}$.

La Organización Mundial de la Salud (OMS) estima que solamente el $63 \%$ de las embarazadas tienen acceso a una visita clínica antenatal y sólo el $42 \%$ de los partos son asistidos por personal de salud entrenado en esta población ${ }^{(7)}$. Los índices de fertilidad son muy altos, no sólo por el desconocimiento de procedimientos anticonceptivos eficaces (los hombres rechazan el preservativo), sino también porque el respeto que recibe una mujer depende en buena parte del número de hijos ${ }^{(4)}$. La comprensión/comunicación entre el personal sanitario y el colectivo de mujeres subsaharianas tiene no sólo la dificultad del encuentro de dos concepciones diferentes sobre salud/enfermedad, además del idioma, ya que por ninguna de las dos partes se dominan lenguas comunes, sino también la derivada de coexistir dos realidades culturales y sociosanitarias bien distintas ${ }^{(19)}$.

Son más frecuentes los embarazos no deseados y es superior la solicitud de interrupciones voluntarias del embarazo, especialmente por motivos sociales y económicos. En el mismo orden de cosas hay una menor utilización de métodos anticonceptivos, generalmente debido a falta de información, no existiendo un rechazo específico. Sería conveniente insistir en las actividades preventivas dentro de este campo, cuidando mucho los aspectos psicosociales de la relación paciente-sanitario por ser éste un tema especialmente delicado ${ }^{(7)}$. En la población subsahariana el acceso a los servicios y el conocimiento de los métodos anticonceptivos son insuficientes, pero esta no es la razón principal por la que continúa siendo una necesidad insatisfecha en la mayoría de los casos. En el oeste y centro de África, la ambivalencia o incluso la hostilidad hacia la anticoncepción es un obstáculo muy serio, pero el problema más general es la preocupación principal por los efectos de salud adversos que sufren las mujeres que utilizan determinados métodos anticonceptivos ${ }^{(20)}$. Éstos son predominantes, especialmente píldoras e inyectables, en esta población inmigrante, siendo muy frecuente la interrupción de estos dos métodos. Por lo general dejan de usarlos al año de utilizarlos entre el $20 \%$ y el $30 \%$ de las mujeres que los utilizan, debido a las preocupaciones sobre sus efectos en la salud 0 a sus efectos secundarios (21). Una de las principales causas del mal cumplimiento se debe a que las mujeres que han utilizado, sin éxito, uno o ambos métodos, no tenían otras opciones en sus países de origen por lo que desconocen el gran abanico de métodos que existen ${ }^{(20)}$.

\section{Europa del Este}

Por lo general las mujeres del Este europeo suelen ser madres con una edad superior a los 23 años y con un tamaño familiar más reducido ${ }^{(4)}$. En este colectivo observamos dos grupos de mujeres según la edad: adultas procedentes de agrupación familiar y jóvenes sin ataduras familiares y con nivel de formación más alto, en situación jurídica en ocasiones ilegal, que deciden buscar mejores condiciones de vida y posibilidad de ampliar sus horizontes profesionales en Europa; son conscientes de las dificultades con que se van a encontrar pero su proyecto de migración es sólido. El concepto salud-enfermedad en lo esencial no es diferente al occidental, aunque no hay 
costumbre de realizar actividades preventivas de forma regular. La solicitud de asistencia sanitaria en España está condicionada por la legalización de su residencia (a veces el paciente no acude al médico por miedo de repatriación), algunos aspectos laborales (por sí su absentismo arriesga su salario o despido por posibles ausencias laborales debidas a los procesos diagnósticos y terapéuticos) y el idioma (en algunas situaciones prefieren esperar para poder ser atendidos en su país). Es costumbre general en estos países el uso de remedios caseros y mucho más peligroso para la vida de la mujer, ya que abundan los métodos tradicionales, hay que tener especial cuidado en lo que a métodos anticonceptivos se refiere ${ }^{(7)}$.

Ni la religión (cristianos católicos y ortodoxos en su mayoría) ni su cultura ofrecen dificultades para la integración. El 14\% de las mujeres que trabajan en la prostitución en España proceden de Europa del Este y unas 10.000 mujeres al año sufren tráfico humano en Bulgaria ${ }^{(7)}$. Los anticonceptivos se utilizan sin dificultades, pero sólo en los últimos años. Las mujeres de Europa del Este utilizan métodos naturales con mayor frecuencia, seguido del DIU. Aunque en general el consumo de anticonceptivos es bajo, las mujeres de Europa del Este conocen bien los métodos anticonceptivos modernos y los aceptan. El aborto provocado es habitual en sus países de origen. En cambio, puede haber más dificultades con respecto a los métodos anticonceptivos con las mujeres rumanas de etnia gitana ya que son un colectivo en el que las tradiciones y costumbres están muy arraigadas ${ }^{(4,7)}$.

Se observa en ellas un bajo consumo de anticonceptivos orales. Después de los métodos naturales, los dispositivos intrauterinos son los más usados. Las mujeres de Europa del Este utilizan los métodos naturales y el DIU como métodos anticonceptivos con mayor frecuencia que los demás, es importante destacar que las mujeres provenientes de estos países (Rusia, Ucrania, Bielorrusia y Rumanía) son la población con mayor índice de IVE, ya que lo consideran un método anticonceptivo más ${ }^{(7,22)}$. El aborto provocado es el principal responsable de la elevada mortalidad materna. En los últimos años, se observa una disminución en las tasas de abortos provocados, y a la vez un aumento en la utilización de los métodos anticonceptivos ${ }^{(7) .}$

Aunque en general el consumo de anticonceptivos es bajo, las mujeres de Europa del Este conocen bien los métodos anticonceptivos modernos y los aceptan, en cambio, puede haber más dificultades en este sentido con las mujeres rumanas de etnia gitana ya que son un colectivo en el que las tradiciones y costumbres están muy arraigadas y suelen rechazar todo tipo de método anticonceptivo ${ }^{(22)}$.

\section{Asia central}

Constituyen el cuarto colectivo de inmigrantes extracomunitarios en España, el 92\% de la población emigrada son de etnia "Han" y procede de la provincia de Zhejiana ${ }^{(4)}$. Tienen un gran respeto por los padres y por los ancianos ya que la familia constituye un pilar primordial de su vida social, añadir que la sumisión de la mujer china ante el hombre ha sido en gran parte superada. Su concepto de enfermedad es muy diferente al occidental, debido a que todo lo basan en la "energía" que circula por canales propios; su exceso (o desbordamiento) o déficit crean las enfermedades ${ }^{(4,7)}$. En general, las inmigrantes asiáticas utilizan los servicios médicos o sociales y comunitarios mucho menos que las inmigrantes provenientes de otras zonas y, por supuesto, que las españolas. Esto se explica por factores culturales como son el hecho de que el paciente asiático suele confiar en remedios naturales 
autodispensables, incluyendo fitoterapia china y masoterapia, además que por lo general son más sufridos ${ }^{(4,7)}$.

También se explica por factores laborales ya que la población asiática suele estar trabajando la mayor parte del día, tienen poco tiempo para acudir al médico; aunque quizás el mayor obstáculo sea el lingüístico, esto es lógico si tenemos en cuenta que la mayoría de ellos tendrían serias dificultades en hacerse entender al explicar su sintomatología, y además estarían muy inseguros pensando si han entendido bien cómo tienen que seguir el tratamiento correctamente. En el caso de los pacientes indocumentados, el problema todavía se acentúa más, ya que en estos casos suelen acudir a médicos que conocen su situación (piensan que no les van a denunciar a la policía, a diferencia de otros facultativos que no les conocen) o a servicios de urgencia de los que tengan alguna referencia; al igual que seguramente ocurra con pacientes de otras etnias, la ignorancia absoluta acerca del funcionamiento de los servicios sanitarios y sociales unido a una dificultad lingüística para poder comunicarse, son limitaciones determinantes a la hora de acceder a dichos servicios. Por ello, es fácil que un paciente asiático, antes de acudir a la consulta con cita previa haya intentado tratamientos caseros 0 medicación despachada por el farmacéutico ${ }^{(4,7)}$.

En Asia no es costumbre acudir al médico si no se padece una patología grave. Por ello cuando a las inmigrantes asiáticas se les efectúa una revisión médica son muy exigentes. Las pacientes chinas y en general todas la asiáticas suelen ser muy directas y tajantes cuando explican sus molestias y enfermedades. Hay distintas opiniones, según diferentes estudios, pero por lo general las inmigrantes asiáticas aceptan bien el tratamiento con anticonceptivos orales o DIU, abortos y demás procedimientos anticonceptivos, aunque anteponen la medicina tradicional china ${ }^{(4,7,22)}$.

\section{Papel de la enfermera}

El personal de Enfermería es un elemento clave en la aplicación, ejecución y continuidad de las prácticas de planificación familiar, siendo fundamental la capacidad y la competencia por parte de la enfermera, ésta es capaz de dar informaciones precisas y responder a las preguntas de los usuarios, contribuyendo a la autonomía de sus clientes; posee suficientes conocimientos técnicos, científicos y culturales, con el fin de atender a la necesidad de servicios de salud sexual y reproductiva de quienes les consultan, incluyendo la capacidad de proporcionar información y comunicarse adecuadamente. Es necesario que los profesionales de salud conozcan las necesidades de las personas que atienden para prestar asistencia en su totalidad, promoviendo la salud y generando la calidad de vida. La enfermera debe orientar, informar y educar al usuario sobre ${ }^{(23)}$ :

- Signos de alarma por los que debe consultar.

- Importancia de las revisiones: las usuarias deben ser informadas sobre la importancia de asistir a consulta para sus controles periódicos para así poder saber si se requiere cambiar el método utilizado en ese momento o localizar posibles complicaciones.

- Prevención de ITS (uso de preservativo como método de protección adicional).

Es necesario que la información proporcionada sea correcta y que las mujeres y las parejas no queden con ninguna duda sobre el método elegido para que se pueda garantizar la eficacia del método anticonceptivo elegido. La eficacia de los métodos 
anticonceptivos depende de la actuación de los profesionales de la salud, incluyendo a la enfermera, pues las informaciones de los diferentes métodos y la forma correcta de transmitirlas están directamente relacionadas con la conquista de la autonomía de mujeres al servicio de la planificación familiar y con la seguridad del uso de los anticonceptivos ${ }^{(23,24)}$.

En definitiva, el papel de la enfermera es proporcionar una buena información para que sea la usuaria la que lleve a cabo la elección del método en función de sus características y preferencias, facilitándoles la posibilidad de resolver dudas acerca del manejo del método, bien programando consultas para valorar la tolerancia a éste o bien disponiendo de personal para resolver las dudas que vayan surgiendo con el uso ${ }^{(25)}$.

\section{CONCLUSIONES}

Las mujeres inmigrantes dependiendo de su nacionalidad hacen diferente uso de los métodos anticonceptivos, debido en parte a los conocimientos insuficientes que poseen, con frecuencia hacen un mal uso de los métodos que utilizan. Según el país de procedencia, tienen diferentes preferencias sobre los métodos anticonceptivos, influidas por sus creencias y costumbres. EI DIU es más utilizado por la inmigrante magrebí y de Europa del Este, el preservativo es el más utilizado por la inmigrante latinoamericana, la asiática prefiere la medicina tradicional china, la subsahariana es la que tiene menor índice de uso de métodos anticonceptivos. No existiendo información actualizada de los métodos empleados por las inmigrantes asiáticas, y la poca que hay es contradictoria, por lo que sería interesante para futuras investigaciones trabajar en esta línea.

La utilización de los métodos anticonceptivos empleados por las mujeres inmigrantes en España en los últimos años ha aumentado, aunque tras esta revisión, queda claro que estas poblaciones necesitan más información adecuada a sus tradiciones y creencias, ya que el uso inadecuado de los métodos o el desconocimiento de mejores alternativas queda patente con esta revisión. Las enfermeras necesitan formación que amplíe sus conocimientos en las diferentes creencias, costumbres y el uso de métodos anticonceptivos de las mujeres inmigrantes según sus nacionalidades, ya que son profesionales claves en la información y orientación de estas mujeres en la consulta de planificación familiar.

Finalmente consideramos, teniendo en cuenta todo lo anterior, que para una buena calidad en la atención de las mujeres inmigrantes en la consulta de planificación familiar por parte de la enfermera, es indispensable que ésta tenga una visión transcultural del cuidado.

\section{REFERENCIAS}

1. González-López, JR. Análisis de las Conductas de Salud de la Población Inmigrante Latinoamericana Adulta de la Ciudad de Sevilla [tesis doctoral]. Departamento de Enfermería, Facultad de Enfermería, Fisioterapia y Podología. Universidad de Sevilla. Sevilla, 2012.

2. Rodríguez Fernández E, Lorca González TM, López Jordan MA, Gómez Moraga A. Mujer inmigrante: motivo de consulta e historia gineco-obstétrica en una zona de salud. Semerg. 2008; 34(2): 59-65. 
3. González-López JR, Rodríguez-Gázquez MA, Lomas Campos MM. Salud Sexual y Reproductiva en América Latina adultos inmigrantes que viven en la ciudad de Sevilla. Enferm. glob. 2013; 12(1): 34-42.

4. Fabre González, E. MIAS: Mujer inmigrante y asistencia sanitaria. Sociedad Española de Ginecología y Obstetricia. Zaragoza: Ed: Saatchi \& Saatchi Healthcare; 2010.

5. Instituto Nacional de Estadística. Resultados detallados: serie 2002-2012: Estimaciones de la población actual (Flujos migratorios estimados) [monografía en Internet]; 2012. [citado 5 Marzo 2014]. Disponible en: http://www.ine.es/jaxiBD/menu.do?L=0\&divi=EPOB\&his $=0 \&$ type $=$ db

6. Ministerio de Sanidad, Servicios Sociales e Igualdad. Interrupción Voluntaria del Embarazo. Datos definitivos correspondientes al año 2012 [monografía en Internet]; 2012. [citado 7 Marzo 2014]. Disponible en:

https://www.msssi.gob.es/profesionales/saludPublica/prevPromocion/embarazo/docs/IVE 2012.pdf

7. Alonso $\mathrm{A}$, Huerga $\mathrm{H}$, Morera J. Manual de atención al inmigrante. $1^{\mathrm{a}}$ Ed. Madrid: Novartis; 2009.

8. España. Real Decreto-ley 16/2012, de 20 de abril, de medidas urgentes para garantizar la sostenibilidad del Sistema Nacional de Salud y mejorar la calidad y seguridad de sus prestaciones. [Internet] Boletin Oficial del Estado, 24 de Julio 2012, núm. 98, pp 31278 [consultados 5 de Marzo de 2014]. Disponible en: http://www.boe.es/boe/días/2012/04/24/pdfs/BOE-A-2012-5403.pdf

9. Terraza Núñez R, Vargas Lorenzo I, Rodríguez Arjona D, Lizana Alcazo T y Vázquez Navarretea ML. Políticas sanitarias de ámbito estatal y autonómico para la población inmigrante en España. Gac Sanit. 2010; 24(2): 115.e1-115.e7

10. Daponte Codina A, Bolivar Muñoz J, Garcia Calvente MM. Las desigualdades sociales en salud. Granada: Escuela Andaluza de Salud Pública; 2008.

11. Instituto Nacional de Estadística. Resultados detallados: Estadística del Padrón Continuo a 1 de enero de 2013 [monografía en Internet]; 2013. [citado 8 Marzo 2014]. Disponible en: http://www.ine.es/jaxi/tabla.do?path=/t20/e245/p04/a2013/l0/\&file=00041008.px\&type=pc axis\&L=0

12. Vicente Torrado, T. La inmigración latinoamericana en España. Expert group meeting on international migration and development in Latin America and the caribbean. Population division. Department of economical and social affaires. United Nations Secretariat: México City; 2005.

13. Jiménez B. Mujeres latinoamericanas en Madrid. Estudios geográficos. 2007; 58 (262): 119- 37.

14. Barona-Vilar C, Más-Pons R, Fullana-Montoro A. Perceptions and experiences of parenthood and maternal health care among Latin American women living in Spain: A qualitative study. Midwifery. 2013; 29 (4): 332-7.

15. Alia MM, Cleland J. Sexual and reproductive behaviour among single women aged 15-24 in eight Latin American countries: a comparative analysis. Soc Sci Med. 2005; 60 (6): 1175-85.

16. Hernando V, Álvarez MC, Arriola L, Arroyo S. Conocimientos y uso de anticonceptivos en la población inmigrante latinoamericana en la Comunidad Autónoma de Madrid. Ministerio de Sanidad y Consumo, Instituto de Salud Carlos III. 2005; 13(4): 37-48.

17. Rodríguez Portilla N.E., Martínez Rojo C. Salud sexual y reproductiva, anticoncepción e interrupción voluntaria del embarazo en las mujeres inmigrantes latinoamericanas. Enferm. glob. 2011; 10 (23): 359-71. 
18. Equipo Trabajo MZC- Chefchaouen, Velasco C, Salas J. Diagnóstico sobre salud sexual y reproductiva en mujeres de la zona rural de Chefchaouen. $1^{\underline{a}} \mathrm{Ed}$. Marruecos: MZC; 2011.

19. Kaplan A. Un estudio sobre la salud sexual y reproductiva en el proceso migratorio de las mujeres migrantes senegambianas. $1^{\underline{a}}$ Ed. Bilbao: Género e Inmigración; 2006.

20. Cleland J, Shah IH. La revolución de los anticonceptivos: todavía son necesarios esfuerzos específicos. Anticonceptivos y derechos reproductivos. Lancet. 2013; 381(9878): 1604-6.

21. Ali MM, Cleland J, Shah $\mathrm{IH}$. Causes and consequences of contraceptive discontinuation: evidence from 60 demographic and health surveys. Geneva: World Health Organization, 2012.

22. Paraíso Torras B, Maldonado del Valle MD, López Muñoz A, Cañete Palomo ML. Anticoncepción en inmigrantes mujeres: Influencia de los aspectos socioculturales de la elección del anticonceptivo método. Medes. 2013; 39(8): 440-4.

23. Moura E, Ferreira S, Costa ML. Conocimiento de enfermería acerca de los métodos anticonceptivos en el contexto del programa de salud de la familia. Enferm. glob. 2010; 9(3): 1-10.

24. Anes A, Diezma JC, Lasheras ML. Los métodos anticonceptivos. Cómo son, cómo actúan, sus ventajas y sus inconvenientes. Comunidad de Madrid. Madrid: Salud Madrid; 2013.

25. Menéndez E, Deza J, Torrents M, Tamargo A, Touris J. Use of contraceptive methods in a family planning clinic. Proc Obstetr y Gine. 2007; 50(12): 675-81.

Recibido: 8 julio 2014; Aceptado: 8 agosto 2014

ISSN 1695-6141

(C) COPYRIGHT Servicio de Publicaciones - Universidad de Murcia 